Supporting Information

\title{
Manipulating External Electric Field and Tensile Strain towards High Energy Density Stability in Fast-charging Li-Rich Cathode Materials
}

Pengfei Liu ${ }^{1}$, Wei He ${ }^{1}$, Yong Cheng ${ }^{1}$, Qian Wang ${ }^{1}$, Chenying Zhang ${ }^{1}$, Qingshui Xie ${ }^{* 1}$, Jiangtao Han ${ }^{1}$,

Zhensong Qiao ${ }^{1}$, Hongfei Zheng ${ }^{1}$, Qun Liu $^{1}$, Laisen Wang ${ }^{1}$, Baihua Qu ${ }^{2}$, Qing Luo ${ }^{1}$, Zi-Zhong Zhu ${ }^{3}$, DongLiang Peng*1 


\section{Experimental Procedures}

Experimental section: Carbonate and oxalate precursors were used to synthesize LLOs. ${ }^{1}$ The former has a smaller lattice constant (S-LLOs) and the latter has a larger one (L-LLOs). They were transformed into oxides first and then mixed with lithium carbonate to get the final product by calcination. Electrodes were made up of LLOs, acetylene black and polyvinylidene fluoride with a mass ratio of 8:1:1 using aluminum foil as a current collector. Meanwhile, the counter and reference electrode were lithium metal, and the separator was Clegard 2350. Cells were assembled in an argon-filled glove box on a condition of $\mathrm{H}_{2} \mathrm{O}<0.1$ p.p.m and $\mathrm{O}_{2}<0.1$

p.p.m. Also, X-ray diffraction patterns, scanning electron microscopy images and transmission electron microscope images were collected on Ultima IV-185 (Rigaku), Su-70 (Hitachi), and Talos F200S (FEI), respectively. Electrochemical tests were conducted on a Neware battery tester with the ambient temperature kept at $30{ }^{\circ} \mathrm{C}$.

Computational section: The pristine structure of $\mathrm{Li}_{1.2} \mathrm{Mn}_{0.54} \mathrm{Co}_{0.13} \mathrm{Ni}_{0.13} \mathrm{O}_{2}$ was adopted from a previous report by using the Vienna ab initio simulation package (VASP).$^{2-4}$ To calculate the electric structure of the deep-delithiation LLOs, all the lithium ions in the pristine structure were removed. In the deep-delithiation LLOs $\mathrm{Mn}_{0.54} \mathrm{Co}_{0.13} \mathrm{Ni}_{0.13} \mathrm{O}_{2}$, the release of lattice oxygen and migration of transition metal ions were not considered for simplicity. Also, all the other details during the calculation can be found in our previous reports. ${ }^{1}$ 


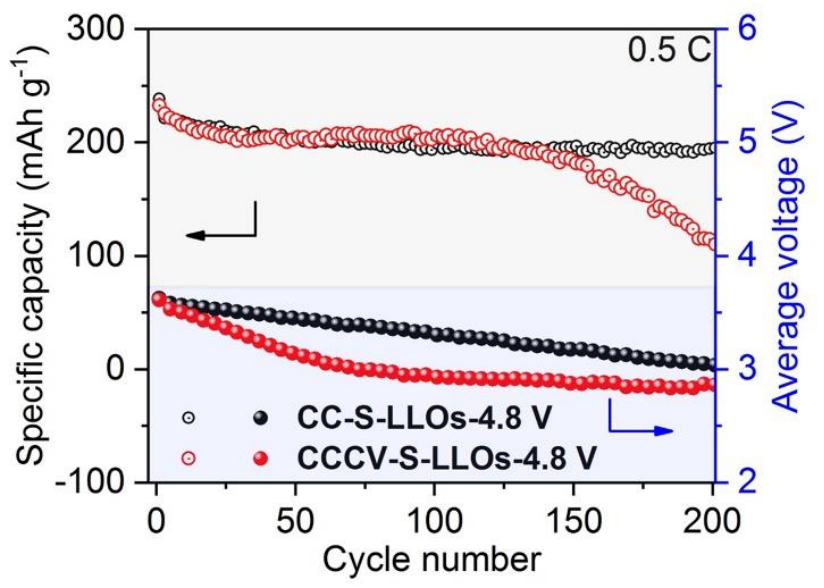

Figure S1. Specific capacities and average voltages at $0.5 \mathrm{C}$ in a voltage range of 2.0-4.8 V. Samples cycled under the CCCV and CV modes have almost the same specific capacity in the first hundreds of cycles, and the sample under the CCCV mode decays faster in the following cycles. However, the CC mode has a greater average voltage than the CCCV mode in almost the entire cycle life. 


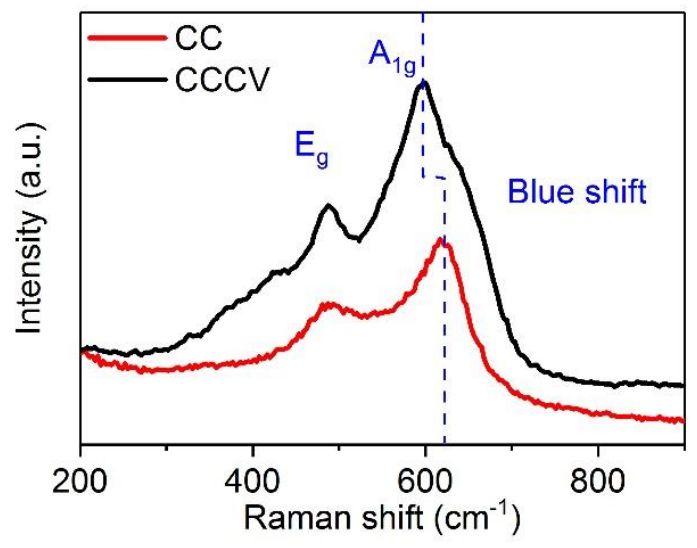

Figure S2. Raman spectra of the samples cycled at $1 \mathrm{C}$ for 200 cycles under different charging modes. Peak $\mathrm{A}_{1 \mathrm{~g}}$ under the $\mathrm{CC}$ mode has a blue shift because of its shortened bond length caused by plenty of transition metal ions in the tetrahedral sites of lithium slabs. ${ }^{5}$ 

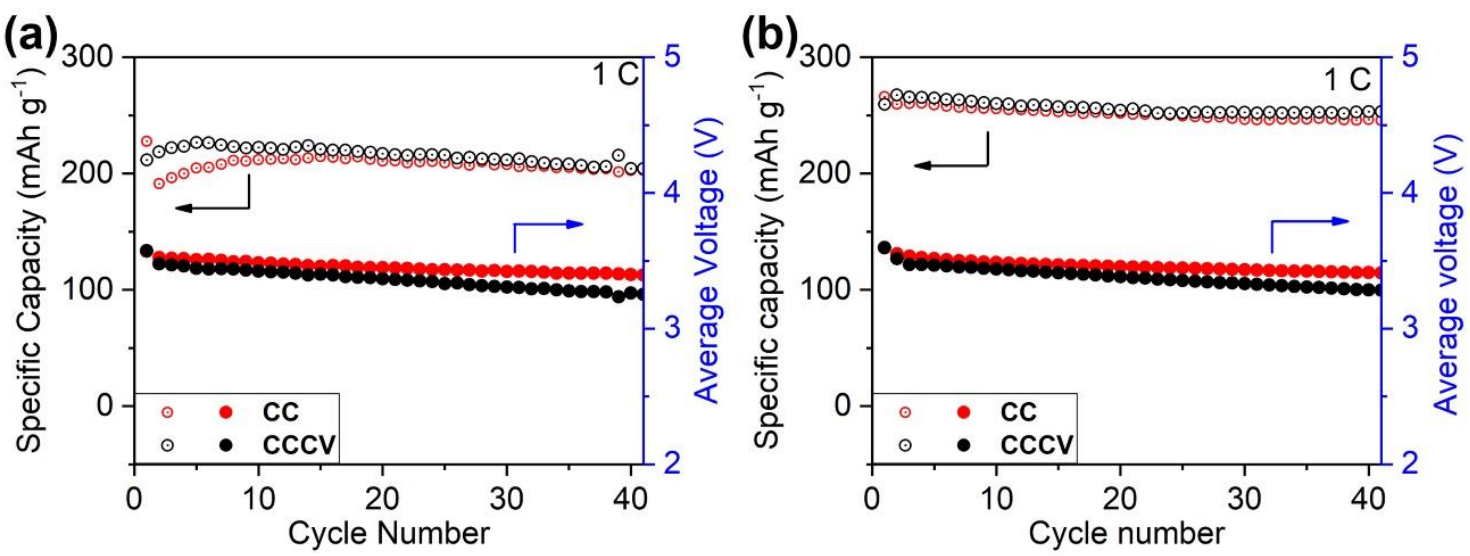

Figure S3. (a,b) LLOs modified by $\mathrm{Na}^{+}$doping (a) and spinel phase coating (b) cycled under CC and CCCV modes ${ }^{6-7}$ For the sample of doping, the specific capacity cycled under CCCV mode decayed faster than that under CC mode despite its acceleration on the activating process. Then, they almost have no obvious difference in specific capacity at the $40^{\text {th }}$ cycle. Meanwhile, the CC mode has a greater average voltage than the CCCV mode. For the sample of spinel phase coating, there is almost no difference in the specific capacity of the two modes, but the CC mode has a greater average voltage than the CCCV mode in all the entire cycling life. The mass loading of active materials was about $1.3 \mathrm{mg} \mathrm{cm}^{-2}$. 

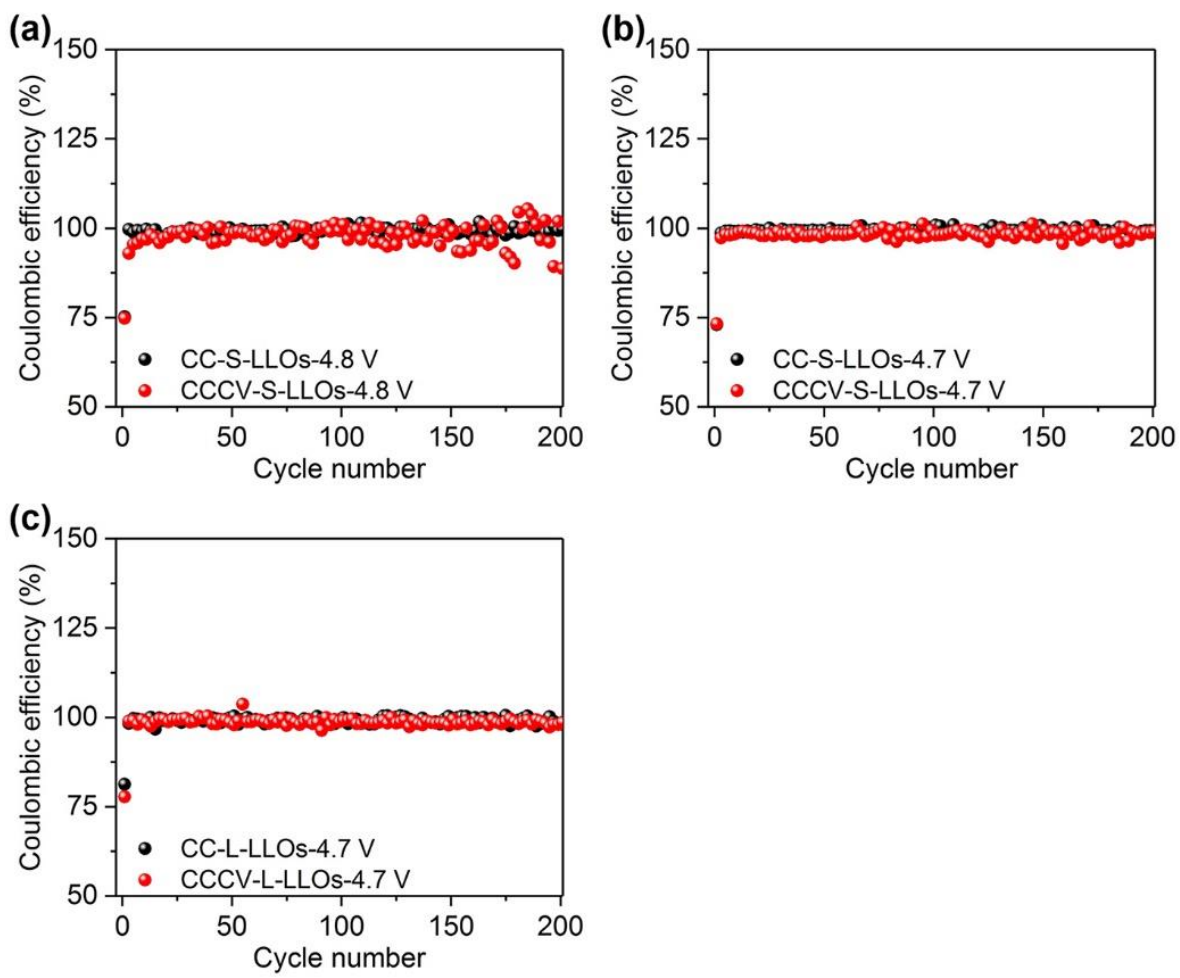

Figure S4. (a-c) Coulombic efficiencies of S-LLOs with a voltage upper limit of $4.8 \mathrm{~V}$ (a), S-LLOs with a voltage upper limit of $4.7 \mathrm{~V}$, and L-LLOs with a voltage upper limit of $4.7 \mathrm{~V}$. All the samples were cycled at $1 \mathrm{C}$ under the $\mathrm{CC}$ and $\mathrm{CCCV}$ modes. Two factors will lead to poor CEs during cycling. One is the external electric field and the other is the dwell time at the CV stage. S-LLOs cycled under the CCCV mode with a voltage upper limit of $4.8 \mathrm{~V}$ have the most chaotic CEs because of the strongest external electric field and the longest dwell time at the CV stage. As indicated in the main text, a strong external electric field will increase the risk of lattice oxygen release and aggravate side reactions. And long dwell time at CV step will accelerate the migration of transition metal ions to lithium slabs, resulting in an unstable lattice and phase transition. Eventually, these two factors will lead to poor CEs during cycling. Therefore, any effort to decrease the influence of these two factors will contribute to improvements on CEs, which has been verified by S-LLOs under the CC mode with a lower voltage upper limit. When the external electric field is weakened and the dwell time at the CV stage is reduced at the same time, L-LLOs under the CCCV mode even has a slight advantage of CEs over L-LLOs under the CC mode. Hence, the method we developed in this paper can improve the CEs of LLOs under the CCCV mode, which is beneficial for its practical application. 


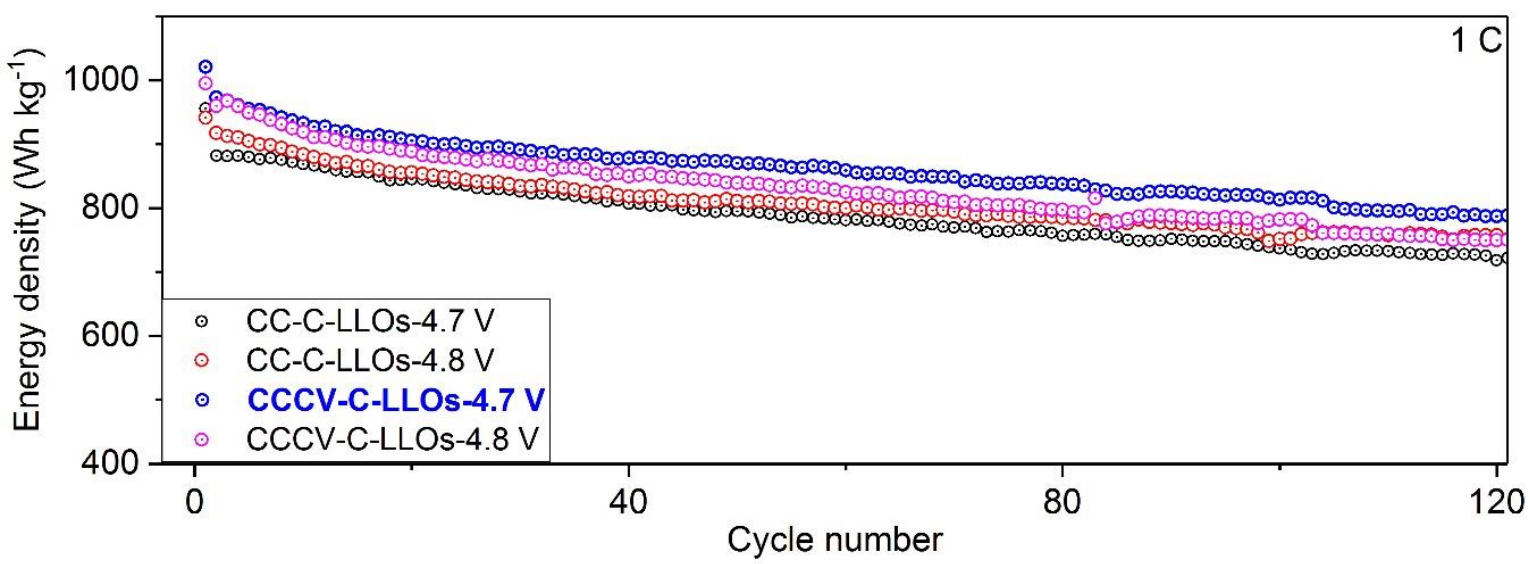

Figure S5. Energy densities of LLOs with surface spinel coating (C-LLOs) under different charging modes. ${ }^{7}$ C-LLOs cycled under the CCCV mode with an upper limit of $4.7 \mathrm{~V}$ have the best energy density, which is in agreement with our conclusion. The mass loading of active materials was about $1.3 \mathrm{mg} \mathrm{cm}^{-2}$. 


\section{Reference}

1. Liu, P.; He, W.; Xie, Q.; Cheng, Y.; Xu, W.; Qiao, Z.; Wang, L.; Qu, B.; Zhu, Z. Z.; Peng, D. L. A Guideline for Tailoring Lattice Oxygen Activity in Lithium-Rich Layered Cathodes by Strain. J. Phys. Chem. Lett. 2019, 10 (9), 2202-2207.

2. Kresse, G.; Joubert, D. From Ultrasoft Pseudopotentials to the Projector Augmented-Wave Method. Phys. Rev. B 1999, 59 (3), 18.

3. Kresse, G.; Furthmüller, J. Efficient Iterative Schemes for Ab Initio Total-Energy Calculations Using a Plane-Wave Basis Set. Phys. Rev. B 1996, 54 (16), 11169-11186.

4. Kresse, G.; Furthmüller, J. Efficiency of Ab-Initio Total Energy Calculations for Metals and Semiconductors Using a Plane-Wave Basis Set. Comput. Mater. Sci. 1996, 6, 36.

5. Zhang, X. D.; Shi, J. L.; Liang, J. Y.; Yin, Y. X.; Zhang, J. N.; Yu, X. Q.; Guo, Y. G. Suppressing Surface Lattice Oxygen Release of Li-Rich Cathode Materials Via Heterostructured Spinel $\mathrm{Li}_{4} \mathrm{Mn}_{5} \mathrm{O}_{12} \mathrm{Coating}$. Adv. Mater. 2018, 30, 1801751.

6. He, W.; Liu, P.; Qu, B.; Zheng, Z.; Zheng, H.; Deng, P.; Li, P.; Li, S.; Huang, H.; Wang, L.; Xie, Q.; Peng, D. L. Uniform $\mathrm{Na}^{+}$Doping - Induced Defects in Li- and Mn-Rich Cathodes for High-Performance Lithium-Ion Batteries. Adv. Sci. 2019, 1802114.

7. Cai, Y.; Ku, L.; Wang, L.; Ma, Y.; Zheng, H.; Xu, W.; Han, J.; Qu, B.; Chen, Y.; Xie, Q.; Peng, D.-L. Engineering Oxygen Vacancies in Hierarchically Li-Rich Layered Oxide Porous Microspheres for High-Rate Lithium Ion Battery Cathode. Sci. China. Mater. 2019, 62 (10), 1374-1384. 